\title{
Diagnosis y nueva descripción de Propanochthus bullifer (Burmeister) (Xenarthra, Glyptodontidae). Consideraciones bioestratigráficas y cronológicas de su procedencia
}

\author{
Martín ZAMORANO
}

División de Paleontología de Vertebrados, Facultad de Ciencias Naturales y Museo, Universidad Nacional de La Plata. Paseo del Bosque s/n, 1900 La Plata. Argentina. CONICET. Cátedra de Zoología Vertebrados; marzamorano@fcnym.unlp.edu.ar

Zamorano, M. 2013. Diagnosis y nueva descripción de Propanochthus bullifer (Bermaister) (Xenarthra, Glyptodontidae). Condideraciones bioestratigráficas y cronológicas de su procedencia. [Diagnosis and new description of Propanochthus bullifer (Burmeister) (Xenarthra, Glyptodontidae). Biostratigraphic and chronological considerations of their origin]. Spanish Journal of Palaeontology, 28 (2), 283-292.

\begin{abstract}
Xenarthra is a diverse group of mammals characteristic of the Neotropical Region. They occur in South America and they are primarily classified into two large groups: Pilosa and Cingulata. The Glyptodontidae are the larger-sized Cingulata group and are recorded from the middle Eocene until the early Holocene; it is noticeable the presence of a dorsal carapace with rigid sutures, in contrast with the other Cingulata (dasypodid and pampatheres). Burmeister recognized Panochthus bullifer, on the basis of fossils from Neogene sediments of the sierras in Córdoba Province. In 1925 Castellanos founded, for this species, the genus Propanochthus, on the ground that the anatomical features of Panochthus bullifer are sufficiently different from the other species of Panochthus. Type material of this species is derived comes from the "Brocherian" of Castellanos. This species was traditionally placed in the "Hoplophorinae" subfamily and "Panochthini" tribe; recent phylogenetic analysis showed that neither of them is a natural group. This contribution provides the first formal diagnosis of Propanochthus bullifer. It also describes the material using a more up-todate and appropriate terminology, comparing it with the
\end{abstract}

\section{RESUMEN}

Burmeister reconoció la especie Panochthus bullifer basándose en restos procedentes de sedimentos neógenos de las sierras de Córdoba. En 1925 Castellanos fundó, para esta especie, el género Propanochthus considerando que los rasgos anatómicos de Panochthus bullifer son suficientemente diferentes a las demás especies de Panochthus. El material tipo de esta especie procede del "Brocherense" de Castellanos. En esta contribución se presenta por primera vez la diagnosis formal de Propanochthus bullifer. Asimismo, se describe el material empleando una terminología más actualizada y apropiada, comparándolo con el de especies afines. El holotipo corresponde a un fragmento de la región posterodorsal de la coraza dorsal y al tubo caudal completo. La ornamentación de los osteodermos de la coraza tiene una gran figura central rodeada por varias hileras (hasta cinco) de figuritas periféricas. La figura central de la zona más anterior es plana y la de la zona posterior convexa, en algunos casos bastante abultada. En los osteodermos del margen posterior, la figura central se ubica caudalmente y está precedida por hasta siete hileras de figuritas. El tubo caudal es más grácil que en Panochthus; es deprimido y su extremo distal es 
related species. The holotype corresponds to a fragment of the postero-dorsal region of the dorsal carapace and the complete caudal tube. The ornamentation of the osteoderms of the carapace has a large central figure surrounded by up to five rows of peripheral small figures. The central figure is flat in the anterior area, convex in the posterior area, and fairly blistered on the margin. In the osteoderms of the posterior margin, the central figure lies caudally, preceded by up to seven rows of small figures. The caudal tube is slightly smaller than in Panochthus; it is depressed and its distal end is notoriously blunt. In dorsal view, the ornamentation consists of flat central figures surrounded by a row (occasionally two rows) of peripheral figures. In lateral view, it has four large sub-elliptical rough figures surrounded by small figures and separated from each other by two opposing smaller subelliptical rough figures. Furthermore, the stratigraphic and geographic distribution of the species is defined, Brochero Formation (Montehermosan-Chapadmalalan [early Pliocenelate Pliocene]) of the Sierras de Córdoba, and the antiquity is discussed.

Keywords: Propanochthus bullifer, Brochero Formation, Neogene, Glyptodontidae, Argentine. notoriamente romo. Dorsalmente su ornamentación está constituida por figuras centrales planas rodeadas de una hilera de figuritas periféricas (excepcionalmente dos); lateralmente, posee cuatro grandes figuras subelípticas rugosas rodeadas por figuritas y separadas entre sí por dos figuras enfrentadas, menores, subelípticas y rugosas. A su vez, se señala la distribución estratigráfica y geográfica, Formación Brochero (Montehermosense-Chapadmalalense [Plioceno tempranoPlioceno tardío]) de las Sierras de Córdoba; también se discute y se aclara la cronológica.

Palabras clave: Propanochthus bullifer, Formación Brochero, Neógeno, Glyptodontidae, Argentina.

\section{INTRODUCCIÓN}

Los xenartros constituyen un muy particular grupo de mamíferos característico de la Región Neotropical, ampliamente representado en el registro fósil de América del Sur, tanto por su extensión temporal como por su frecuencia de registros (Paula Couto, 1979; ScillatoYané, 1982; Wetzel, 1982; Scillato-Yané \& Carlini, 1995; Scillato-Yané et al., 1995). El grupo incluye 31 especies vivientes agrupadas en 14 géneros (Wetzel, 1985; Redford \& Eisenberg, 1992; McKenna \& Bell, 1997; Aguiar \& da Fonseca, 2008). Los Xenarthra se dividen, principalmente, en dos grandes grupos: Pilosa y Cingulata.

Los Cingulata poseen caracteres esquelético-dentarios que los distinguen claramente del resto de los Xenarthra (ver Hoffstetter, 1982; Patterson et al., 1989; Gaudin, 1999; McDonald, 2003), así como caracteres moleculares (de Jong et al., 1985; Sarich, 1985; Delsuc et al., 2002, 2003), y es la presencia de una coraza bien desarrollada formada por placas óseas dérmicas (osteodermos) la característica más evidente para diferenciarlos (Wetzel, 1985). Los Cingulata son los xenartros más antiguos en el registro y están representados por dos grupos principales: 1) Dasypodoidea (con representantes actuales y extintos) y 2) Glyptodontoidea (con representantes extintos de Glyptodontidae, Palaeopeltidae y Pampatheriidae) (ver McKenna \& Bell, 1997).

Los Glyptodontidae son el grupo de Cingulata de mayor tamaño (Scillato-Yané \& Carlini, 1998; Fariña,
2001), pudiendo superar los $2.300 \mathrm{~kg}$ (Soibelzon et al., 2012), y se registran desde el Eoceno medio hasta el Holoceno temprano (Scillato-Yané, 1976; Fernicola, 2008; Soibelzon et al., 2012). Lo más notorio entre los Glyptodontidae es que la coraza dorsal está compuesta por osteodermos articulados por suturas rígidas en toda la extensión del dorso, sin presentar en la región intermedia (o en la parte anterior e intermedia) las bandas transversales continuas móviles, típicas de dasipodódidos y pampatéridos (ver Ameghino, 1889; Fariña, 1985; Ferigolo, 1985; Fariña \& Vizcaíno, 2001; Croft et al., 2007; Zurita, 2007; Fernicola, 2008; Zamorano, 2012a). Una de las clasificaciones tradicionales más aceptadas divide la familia en cinco subfamilias (Glyptatelinae, Propalaehoplophorinae, Doedicurinae, Glyptodontinae y Hoplophorinae) (Hoffstetter, 1958).

La subfamilia "Hoplophorinae" se registra desde el Mioceno medio hasta el Holoceno temprano, su característica más notoria es la precoz adquisición de un tubo caudal (Zurita, 2007; Zamorano, 2012a). No obstante, esta subfamilia no es considerada un grupo natural (Fariña, 1993; Fernicola, 2008; Porpino et al., 2010). "Hoplophorinae" alberga siete tribus sensu Hoffstetter, 1958: Palaehoplophorini, Neuryurini, Neothoracophorini, Plohophorini, Hoplophorini, Lomaphorini y Panochthini.

La Tribu "Panochthini” (Plioceno-Pleistoceno tardío) incluye los géneros Nopachtus Ameghino, 1888, Propanochthus Castellanos, 1925 y Panochthus Burmeister, 1866 y un género dudoso del Pleistoceno 
de Brasil: Parapanochthus Moreira, 1965, 1971; estos gliptodóntidos son de mediano a gran tamaño (Zamorano, 2012a). Este no corresponde a un grupo natural (Zamorano, 2012a, 2012b; Zamorano \& Brandoni, 2012, en prensa).

Burmeister (1874) reconoció la especie Panochthus bullifer, a base de restos procedentes de sedimentos neógenos de las Sierras de Córdoba (Fig. 1). En 1925 Castellanos fundó, para esta especie, el género Propanochthus, considerando que los rasgos anatómicos de Panochthus bullifer son suficientemente diferentes a las demás especies de Panochthus. También agrega que con el reconocimiento de su nuevo género, observando la ornamentación del tubo caudal, se puede señalar una serie evolutiva, Nopachtus, Propanochthus, Panochthus (Castellanos, 1925, p. 96). Luego, Castellanos (1942a) describe el holotipo con más detalle que Burmeister (1874); sin embargo, no existe ninguna diagnosis formal de la especie. El material tipo de esta especie procede del "horizonte Brocherense" de Castellanos (1942a, 1942b, 1944); ninguno de los anteriores autores brinda una procedencia estratigráfica precisa. Esta especie es uno de los fósiles guías de la Biozona de Asociación de Nonotherium hennigi-Propanochthus bullifer, propuesta por Cruz (2011) para la Provincia de Córdoba, referida al Montehermosense-Chapadmalalense (Plioceno temprano-Plioceno tardío) y correlacionable tentativamente con las biozonas de Trigodon gaudryi, Neocavia depressidens y Paraglyptodon chapadmalensis de la Provincia de Buenos Aires (Cruz, 2011).

En esta contribución se presenta por primera vez la diagnosis formal de Propanochthus bullifer. Asimismo, se describe el material empleando una terminología más actualizada y apropiada, comparándolo con el de especies afines. A su vez se discute sobre los estratos de los cuales procede este material.

\section{MATERIALES Y MÉTODOS}

Los materiales asignados a Propanochthus bullifer se encuentran depositados en: Sección Paleontología Vertebrados, Museo Argentino de Ciencias Naturales "Bernardino Rivadavia" (MACN), Colección Paleovertebrados (Pv), Ciudad Autónoma de Buenos Aires, Argentina; Museo Universitario Florentino y Carlos Ameghino (MUFyCA), Rosario, Argentina. Los materiales de comparación están en: Museo de la Plata, División Paleontología Vertebrados, Facultad de Ciencias Naturales y Museo, Universidad Nacional de La Plata (MLP), La Plata, Argentina; Museo Nacional, Rio de Janeiro (MNRJ), Rio de Janeiro, Brasil.

Para la descripción del material se siguió a Porpino \& Bergqvist (2002) y a Zamorano (2012a). Para la sistemática se optó por la propuesta de Zamorano \& Brandoni (en prensa).

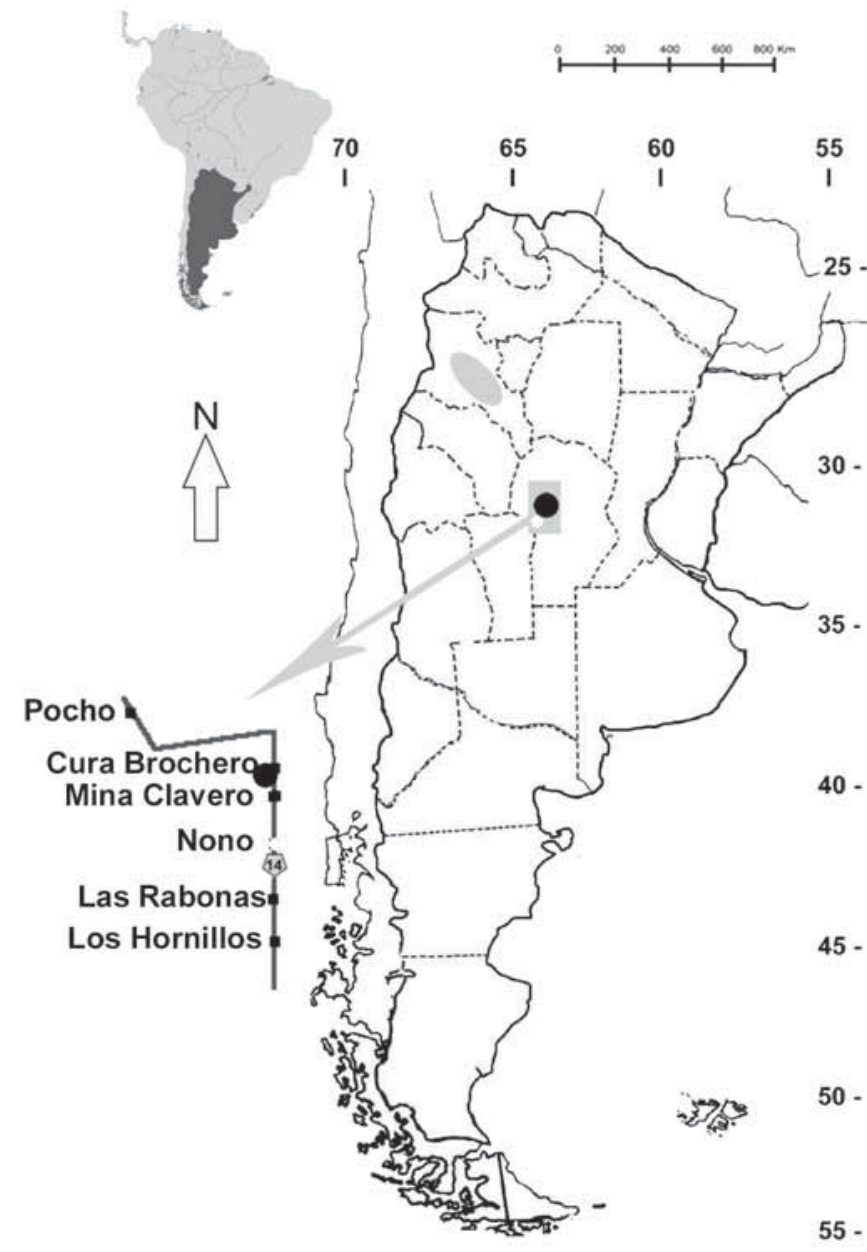

Figura 1. Distribución geográfica de Propanochthus bullifer. Círculo negro, holotipo (cercanías de Villa Cura Brochero, Sierras de Córdoba); círculo blanco, localidad con información precisa de registros, Nono (MUFyCA 370); sombreado gris, distribución generalizada sobre la base de datos éditos y de catálogos (Sierras de Córdoba, Catamarca).

Geographic distribution of Propanochthus bullifer. Black circle, holotype (Villa Cura Brochero near, Sierras de Córdoba); white circle, location with accurate information of records, Nono (MUFyCA 370); shaded in grey, generalized distribution on the basis of data edits and catalog (Sierras de Córdoba, Catamarca).

Las medidas que se utilizaron para el tubo caudal (Fig. 2), única estructura anatómica completa, fueron: diámetro dorso-ventral del tubo (DDV), a nivel a) distal, b) medio, c) proximal; diámetro transverso del tubo (DT), a nivel a) distal, b) medio, c) proximal; longitud máxima (LM); número de figuras laterales (incluyendo la más distal [= terminal]) (NFL), a) derecho, b) izquierdo. 


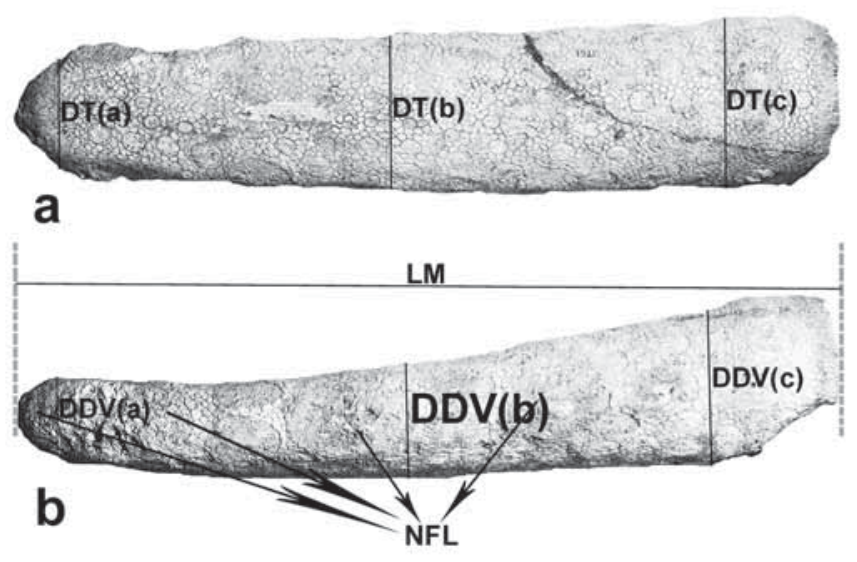

Figura 2. Medidas realizadas en el tubo caudal de Propanochthus bullifer. a) diámetro transverso (DT), a nivel (a) distal, (b) medio, (c) proximal. b) diámetro dorso-ventral (DDV), a nivel (a) distal, (b) medio, (c) proximal. Measurements in the caudal tube of Propanochthus bullifer. a) transverse diameter (TD), at the (a) distal, (b) medium, (c) proximal. b) dorso-ventral diameter (DDV), at the (a) distal, (b) medium, (c) proximal.

\section{SISTEMÁTICA}

Magnorden XENARTHRA Cope, 1889

Orden CINGULATA Illiger, 1811

Suborden GLYPTODONTIA Ameghino, 1889

Superfamilia GLYPTODONTOIDEA Gray, 1869

Familia Glyptodontidae Gray, 1869

Género Propanochthus Castellanos, 1925

(Figs 3-4)

1874 Panochthus Burmeister, p. 5-6, 103, 148-155, 224, 405, Pl. XV1, Figs 3-5.

Especie tipo. Propanochthus bullifer (Burmeister, 1874) [originalmente como Panochthus].

Diagnosis. Osteodermos de la región postero-dorsal de la coraza dorsal con figura central, plana o convexa, rodeada por entre tres y siete hileras de figuritas periféricas, a diferencia de Nopachtus que tiene una o dos, y similarmente a Panochthus intermedius que también posee hasta siete. Tubo caudal más grácil que en Panochthus, deprimido dorso-ventralmente, elevado en el extremo distal, el cual es notoriamente romo; ornamentación dorsal con figuras centrales planas rodeadas de una hilera de figuritas periféricas (excepcionalmente dos); hay cuatro figuras laterales, subelípticas, de similar tamaño y menos rugosas que en Panochthus, rodeadas por figuritas y separadas entre sí por dos figuras enfrentadas, menores, subelípticas y rugosas.
Distribución estratigráfica y geográfica. Formación Brochero (Montehermosense-Chapadmalalense [Plioceno temprano-Plioceno tardío] [ver Cruz, 2013]) de las Sierras de Córdoba, en las cercanías de Villa Cura Brochero. También existen registros no verificables en otras regiones geográficas.

Especies asignadas. Propanochthus bullifer (Burmeister, 1874).

Propanochthus bullifer (Burmeister, 1874)

(Figs 3-4)

1874 Panochthus bullifer Burmeister, p. 5-6, 103, 148155, 224, 405, Pl. XV1, Figs 3-5.

Diagnosis. La misma que para el género (única especie conocida).

Holotipo. MACN-Pv 1761, fragmento de la región postero-dorsal de la coraza dorsal, incluido parte del borde posterior, y tubo caudal y, un pie anterior derecho casi completo con cúbito y radio, una porción posterior de la pelvis y las cinco primeras vértebras caudales (extraviados).

Procedencia estratigráfica y geográfica. Formación Brochero (Montehermosense-Chapadmalalense [Plioceno temprano-Plioceno tardío] [ver Cruz, 2013]) de las Sierras de Córdoba, en las cercanías de Villa Cura Brochero.

Material. El holotipo y MUFyCA 370, osteodermos sueltos de la coraza dorsal. Estos materiales fueron asignados por Castellanos (1942a, 1944) a Nopachtus coagmentatus (ver en Cruz, 2011, 2013, descripción y figuras de este material). Este último material se diferencia claramente de la diagnosis de $N$. coagmentatus (ver Zamorano et al., 2011, p. 64) y se ajusta a la de $P$. bullifer.

Distribución estratigráfica y geográfica. La misma que para el género (única especie conocida).

Descripción. Coraza dorsal. Los osteodermos preservados corresponden a la región postero-dorsal. Su ornamentación consiste en una gran figura central rodeada por varias hileras (hasta cinco) de figuritas periféricas (Fig. 3a). Estas figuritas son pentagonales o hexagonales (Fig. 3b), semejantes a las que rodean la figura central en las especies de Nopachtus, en las cuales están rodeadas hasta por dos hileras. En Propanochthus bullifer, la figura central de los osteodermos de la zona más anterior de la región preservada es plana, como en la región anterior de la coraza de Nopachtus cabrerai. La figura central, en los osteodermos cercanos a la escotadura caudal, es convexa, 


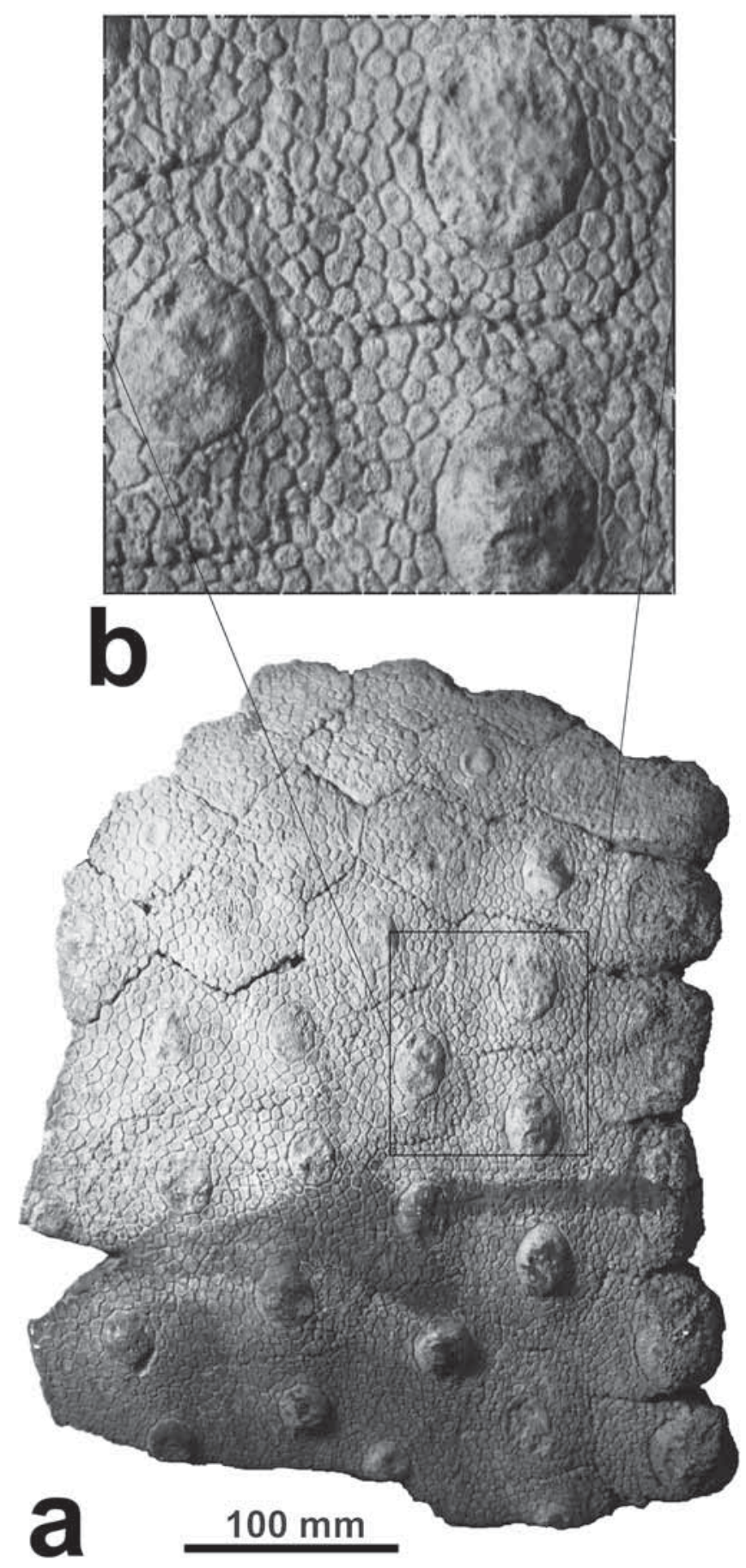

Figura 3. Propanochthus bullifer, holotipo (MACN-Pv 1761). a) fragmento de la región postero-dorsal de la coraza. b) detalle de osteodermos.

Propanochthus bullifer, holotype (MACN-Pv 1761). a) fragment of the postero-dorsal region of the carapace. $\boldsymbol{b})$ detail of osteoderms. en algunos casos bastante abultada, como en la región posterior de la coraza de Nopachtus cabrerai. Las hileras de figuritas periféricas son numerosas, de tres a siete, a diferencia de las especies de Nopachtus, Phlyctaenopyga y Stromaphorus, que poseen de una a dos hileras. En los osteodermos del margen posterior, la figura central se ubica caudalmente, y está precedida por hasta siete hileras de figuritas.

Tubo caudal. Presenta menor tamaño y es más grácil que en las especies de Panochthus (Tabla 1). Es deprimido dorso-ventralmente, aunque su sección proximal, como sucede en Panochthus, es más circular, disminuyendo su diámetro dorso-ventral en sentido distal. Al igual que en Panochthus, el tubo es dorsalmente cóncavo con la región distal más elevada. Su extremo distal es notoriamente romo (Fig. 4). Castellanos (1942a, p. 476) dice: “es un espécimen viejo y se halla gastada la parte apical”. Aunque por el desgaste el extremo distal del tubo caudal se ve extremadamente romo, no se duda en que dicho extremo sea marcadamente romo. El desgaste (seguramente por rodamiento) que se aprecia no afecta a ninguno de los caracteres diagnósticos del tubo.

Tabla 1. Medidas (en mm) del tubo caudal de Propanochthus bullifer, Panochthus tuberculatus (MLP 16-32), Pa. subintermedius (MACN-Pv 5130), Pa. greslebini (MNRJ 2760/1V) y Pa. jaguaribensis (MNRJ 2759/1V). Measurements (in $\mathrm{mm}$ ) of caudal tube of Propanochthus bullifer, Panochthus tuberculatus (MLP 16-32), Pa. subintermedius (MACN-Pv 5130), Pa. greslebini (MNRJ 2760/1V) and Pa. jaguaribensis (MNRJ 2759/1V).

\begin{tabular}{lcccccccccc}
\hline & LM & DT & DT & \multicolumn{1}{c}{ DT } & DDV DDV DDV NFL NFL \\
& & a) & b) & c) & a) & b) & c) & a) & b) \\
\hline $\begin{array}{l}\text { Propanochthus } \\
\text { bullifer }\end{array}$ & 773 & 97 & 129 & 156 & 78 & 107 & 145 & 4 & 4 \\
$\begin{array}{l}\text { Panochthus } \\
\text { tuberculatus }\end{array}$ & 940 & 110 & 179 & 168 & 87 & 120 & 186 & 4 & 4 \\
$\begin{array}{l}\text { Panochthus } \\
\text { subintermedius }\end{array}$ & 960 & 99 & 153 & 198 & 82 & 109 & 201 & 5 & 6 \\
$\begin{array}{l}\text { Panochthus } \\
\text { greslebini }\end{array}$ & 774 & 100 & 197 & 180 & 78 & 105 & 146 & 4 & 4 \\
$\begin{array}{l}\text { Panochthus } \\
\text { jaguaribensis }\end{array}$ & 985 & 97 & 159 & 172 & 80 & 108 & 156 & 5 & 5 \\
\hline
\end{tabular}

En vista dorsal (Fig. 4a) se observa que la ornamentación que cubre totalmente la superficie es ligeramente comparable a la presente en la coraza dorsal; hay figuras centrales rodeadas por hileras de figuritas periféricas, aunque en este caso solamente por una hilera (pocas veces dos) rodeando la central. Todas las figuras centrales de la cara dorsal son planas, nunca son convexas como 
en la coraza dorsal, ni rugosas como en algunas de las observadas en esta vista en Panochthus.

En vista ventral (Fig. 4b) la ornamentación es similar a la de la cara dorsal, con las figuras centrales levemente mayores.

En vista lateral (Figs 4c-4d), desde la zona más proximal del tubo, se ven dos figuras rugosas subcirculares, seguidas, hacia el extremo distal, por cuatro figuras subelípticas rugosas más grandes (más del triple) y deprimidas (Tabla 1). Dichas figuras son de tamaño similar, pero menos rugosas y deprimidas que en Panochthus; las grandes cuatro figuras se encuentran rodeadas por varias hileras de figuritas y entre ellas hay un par de figuras subelípticas rugosas de menor tamaño enfrentadas ("par dorso-ventral”, según Porpino \& Bergqvist, 2002, para Panochthus grelebini y $P$. jaguaribensis), ubicadas una en el margen dorsal y la otra en el margen ventral.

Comentarios. Además de la coraza dorsal y el tubo caudal ya mencionados, el holotipo de $P$. bullifer está conformado por: “....un pie anterior derecho casi completo con cúbito y radio; una porción posterior de la pelvis y las cinco primeras vértebras caudales" (Castellanos, 1942a, p. 468), brevemente descriptos por Burmeister (1874) y Castellanos (1942a); pero estos materiales están extraviados.

Según Castellanos (1942a, p. 484-486): (1) las vértebras caudales son las cinco más proximales de las que se encuentran dentro del tubo caudal, y resultan más pequeñas que en Panochthus. Según Burmeister (1874), habría 6 ó 7 más, con lo que Propanochthus bullifer tendría un total de 11 ó 12 vértebras caudales; en cambio, Panochthus tuberculatus tiene 14, 6 proximales y 8 dentro del tubo (ver esqueleto MLP 16-29, que coincide con la estimación de Castellanos, 1942a, p. 484); (2) el fragmento de pelvis y de sinsacro conservado consiste, según Burmeister (1874, p. 154), "en el arco sacral libre con las dos apófisis transversas de la última vértebra, que se unen con la ala ciática perpendicular" (sic). Aunque la descripción es confusa, se deduce que ha de tratarse de parte del sinsacro fusionado con parte del isquion. Estos huesos son más pequeños y gráciles que los de $P$. tuberculatus, según Burmeister (1874) y Castellanos (1942a); (3) la ulna y el radio son más cortos y gráciles que en Panochthus tuberculatus (en MACN-Pv 1761, según Burmeister, 1874, con LM: $265 \mathrm{~mm}$ y $151 \mathrm{~mm}$, respectivamente); (4) los dedos son cuatro (que corresponden al II, III, IV y V) y tienen una longitud similar a los homólogos de $P$. tuberculatus.

Kraglievich (1934) menciona el hallazgo, en el Puerto de La Plata, de Panochthus bullifer. Pero este resto fue identificado por Ameghino y él confundía la especie citada con $P$. intermedius. Además, no se figura ni se indica dónde se conserva dicho material; de tal manera, esta asignación no puede comprobarse.

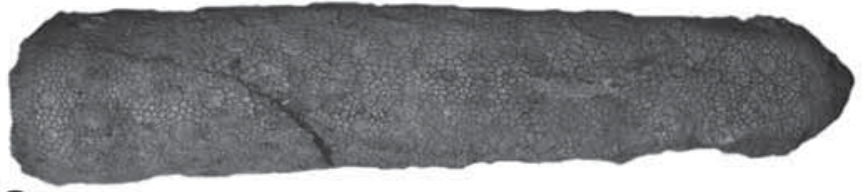

a

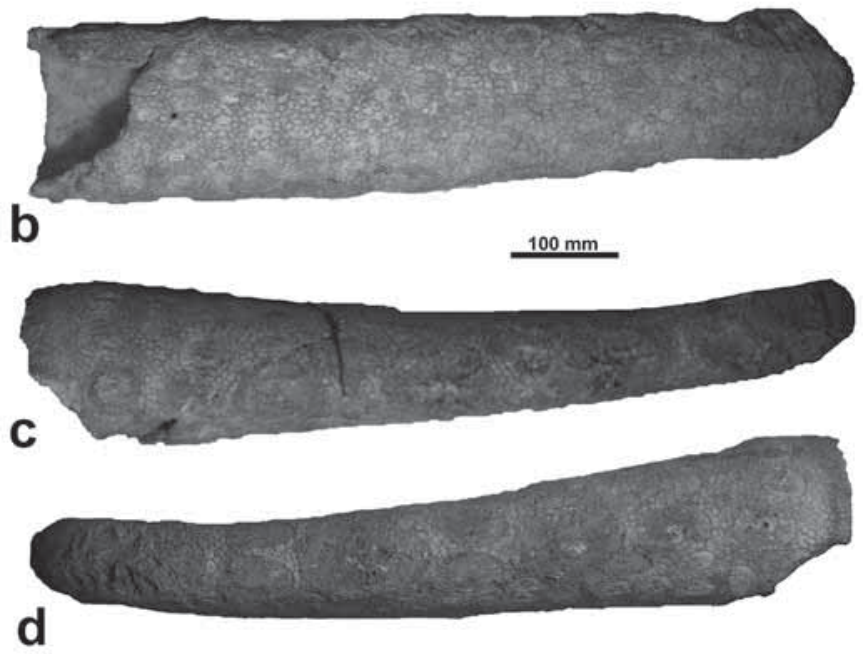

Figura 4. Propanochthus bullifer, holotipo (MACN-Pv 1761). Tubo caudal: a) vista dorsal. b) vista ventral. c) vista lateral izquierda. d) vista lateral derecha. Propanochthus bullifer, holotype (MACN-Pv 1761). Caudal tube: a) dorsal view. b) ventral view, c) left lateral view, d) right lateral view.

\section{DISCUSIÓN}

En las últimas propuestas filogenéticas de Glyptodontidae ha quedado demostrado que Hoplophorinae no es un grupo natural (Fariña, 1993; Fernicola, 2005, 2008; Porpino et al., 2009, 2010; Fernicola \& Porpino, 2012) y que tampoco lo es Panochthini (Zamorano, 2012a, 2012b; Zamorano \& Brandoni, 2012, en prensa). Zamorano (2012a, 2012b) y Zamorano \& Brandoni (2012, en prensa) como resultado de sus análisis filogenéticos relacionan a Propanochthus bullifer, Nopachtus coagmentatus y $N$. cabrerai en un clado que agrupa también a Stromaphorus compressidens y Phlyctaenopyga ameghini (ambas "Plohophorini”); dejando a Panochthus en un clado diferente. En la sistemática más acatada por la comunidad científica, Propanochthus, Nopachtus y Panochthus son géneros tradicionalmente ubicados como un grupo, "Panochthini" (Castellanos, 1942a; Hoffstetter, 1958; Cattoi, 1966; Paula Couto, 1979; Scillato-Yané et al., 1995; Scillato-Yané \& Carlini, 1998; McKenna \& Bell 1997; Zamorano, 2005; Zamorano \& Scillato-Yané, 2006; Zurita, 2007; Zamorano et al., 2011; Zurita et al., 2011; Zamorano, 2012a, 2012b; Zamorano \& Brandoni, 2012, en prensa). En el apartado "SISTEMÁTICA" se coloca a Propanochthus inmediatamente abajo de la Familia Glyptodontidae y no 
se incluye ni la Subfamilia "Hoplophorinae” Huxley, 1864, ni la Tribu "Panochthini" Castellanos, 1927.

No es poca la controversia existente en cuanto a la procedencia geográfica de los materiales referidos a Propanochthus bullifer. Castellanos (1942a, p. 468470) comenta detalladamente esta polémica. Aquí la mencionaremos sintéticamente (ver Fig. 1): (1) En 1865 el Director del proceso de amalgamación del Ingenio de Pilciao de la Provincia de Catamarca, Sr. Federico Schickendantz, le envió a Burmeister fragmentos del esqueleto y de la coraza de un gliptodonte de gran tamaño hallado a 20 leguas (sic) [96 km] al noreste del pueblo de Pilciao, en el valle del Río Belén a 1.524 msnm (expresados en pies en la publicación original, 5.000). Burmeister atribuyó, en un principio, este material a Panochthus tuberculatus, pero luego lo asignó a $P$. bullifer (Burmeister, 1874, p. 149-150). Se desconoce dónde se encuentra depositado este material y tampoco está figurado. (2) En 1869, en terrenos de propiedad del Sr. Arístides Ramallo, se halló el tubo caudal y una porción de coraza dorsal en el Valle El Transito [actualmente Villa Cura Brochero] a $610 \mathrm{msnm}$ [2.000 pies según Burmeister], entre la Sierra de Achala y el cordón de Achalita. Al considerar este material, Burmeister decidió atribuir el tubo caudal y la porción de coraza dorsal a Panochthus bullifer (Burmeister, 1874, p. 150). Este material está depositado en el MACN, MACNPv 1761, y es el holotipo de la especie. (3) Ameghino (1885, p. 348) menciona fragmentos de coraza dorsal hallados en las colecciones del Museo Antropológico y Paleontológico de la Universidad Nacional de Córdoba (fundado por él mismo en 1885). Se desconoce dónde se encuentra depositado este material y tampoco está figurado. (4) Por último, Burmeister (1879, p. 417) manifiesta que solamente conoce dos corazas incompletas de Panochthus bullifer: la de Catamarca, "(1)”, y la de Córdoba, “(2)”.

En tanto que, el material MUFyCA 370 procede de la citada Formación Brochero de las barrancas que están entre las de Catedral y El Divisadero, margen derecha del río Grande de Nono, Valle de Traslasierra, Nono, Córdoba, Argentina (ver Cruz, 2011).

La procedencia bioestratigráfica tampoco es clara. Los sedimentos asignados al "horizonte Brocherense" de Castellanos (1942a, 1942b y 1944) afloran en el fondo de los grandes valles intermontanos de las Sierras de Córdoba, especialmente en los Valles de San Alberto y Calamuchita (Cruz, 2011). La edad de estos sedimentos ha sido inferida por registros de fósiles de mastofauna. Castellanos (1942b) nominó este "horizonte" por la asociación de Nonotherium hennigi (Toxodontia), Paedotherium brocherense (Typotheria) y Nopachthus coagmentatus (sic), cuyo material halló en el Valle de San Alberto (localidades de Villa Cura Brochero y Nono). También incluyó en la misma asociación registros de otros mamíferos provenientes de Córdoba, Paraglyptodon cordubensis y Propanochthus bullifer, aunque estos últimos carecen de procedencia estratigráfica y geográfica precisa. Estos registros hicieron que Castellanos proponga que este "horizonte" se ubique entre el Chapadmalalense y el Ensenadense (ver Castellanos 1942b, 1944, 1951, 1956, 1958). Para Marshall et al. (1984) y Cione \& Tonni (1995) los niveles contenían una mezcla de taxones montehermosenses y ensenadenses. Luego, Bonalumi et al. (1999) asignó estos sedimentos, junto con otros suprayacentes, al Plioceno temprano-Plioceno tardío temprano. Para Gaido et al. (2006) dichos sedimentos corresponden al Plioceno temprano-medio (ver Cruz, 2011). En tanto que para Tauber \& Álvarez (2003) y Zurita (2007) corresponden al lapso Mioceno tardío-Plioceno.

\section{CONCLUSIONES}

La validez de Propanochthus bullifer está basada en la siguiente combinación de caracteres: presenta en los osteodermos de la región postero-dorsal una notable figura central, plana o convexa (hacia el margen posterior esta es muy "ampollada”), rodeada por entre tres y siete hileras de figuritas periféricas muy pequeñas.

Si bien Propanochthus bullifer está más relacionada con los "Plohophorini" su tubo caudal es muy similar al de Panochthus. Asimismo, los rasgos de los osteodermos de la coraza se asemejan más a los de Nopachtus, Phlyctaenopyga y Stromaphorus.

La procedencia estratigráfica y geográfica del holotipo es: Formación Brochero (Montehermosense - Chapadmalalense [Plioceno temprano-Plioceno tardío]) de las Sierras de Córdoba, en las cercanías de Villa Cura Brochero.

\section{AGRADECIMIENTOS}

A Gustavo J. Scillato-Yané y Alfredo E. Zurita por el apoyo y la revisión general. Alfredo A. Carlini, Daniel Perea y Juan C. Fernicola por la revisión de la descripción anatómica. A Eduardo P. Tonni y Alberto L. Cione por los comentarios sobre la cronología y biostratigrafía. A Laura E. Cruz por facilitarme su Tesis Doctoral. A Esteban Soibelzon y Flávio Góis por las fotografías. A Julio Aguirre, un revisor anónimo y Richard A. Fariña por los enriquecedores arbitrajes.

\section{REFERENCIAS}

Aguiar, J.M. \& Fonseca, G.A.B. da. 2008. Conservation status of the Xenarthra. In: The Biology of the Xenarthra (eds. Vizcaíno, S.F. \& Loughry, W.J.). University Press of Florida, Gainesville, 215-231. 
Ameghino, F. 1885. Nuevos restos de mamíferos fósiles oligocenos recogidos por el Profesor Pedro Scalabrini y pertenecientes al Museo Provincia de la ciudad de Paraná. Boletín de la Academia Nacional de Ciencias, 8, 5-207.

Ameghino, F. 1888. Rápidas diagnosis de mamíferos fósiles nuevos de la República Argentina. Obras Completas, 5, 471-480.

Ameghino, F. 1889. Contribución al conocimiento de los mamíferos fósiles de la República Argentina. Actas Academia Nacional de Ciencias de Córdoba, 6, 1-1027.

Bonalumi, A., Martino, R., Baldo, E., Zarco, J., Sfragulla, J., Carignano, C., Kraemer, P., Escayola, M., Tauber, A., Cabanillas, A., Juri, E. \& Torres, B. 1999. Hoja Geológica 3166-IV, Villa Dolores. Provincias de Córdoba, La Rioja y San Luis. Instituto de Geología y Recursos Minerales, Servicio Geológico Minero Argentino, Buenos Aires. Boletín 250, 123 pp.

Burmeister, G. 1866. Lista de los mamíferos fósiles del terreno diluviano. Anales del Museo Público de Buenos Aires, 1, 121-232.

Burmeister, G. 1874. Monografía de los Glyptodontes en el Museo Público de Buenos Aires. Anales del Museo Público de Buenos Aires, 2, 1-412.

Burmeister, G. 1879. Description physique de la République Argentine d'après des observations personnelles et étrangères. In: Tome troisiéme: Animaux vertébrés. Première partie: mammiféres vivants et éteints (ed. PaulEmile Coni), Buenos Aires.

Castellanos, A. 1925. Breves notas sobre evolución de la coraza y del extremo caudal en los Loricata (=Hicanodonta)". Revista de "El Circulo", número extraordinario, 93-96.

Castellanos, A. 1927. Descripción de un fragmento de tubo caudal de un nuevo daedicurino en relación con sus géneros afines. Anales del Museo de Historia Natural de Montevideo, 2, 265-300.

Castellanos, A. 1942a. A propósito de los géneros Plohophorus, Nopachthus y Panochthus (3a. parte). Publicaciones del Instituto de Fisiografía y Geología de la Universidad Nacional del Litoral, 11, 413-592.

Castellanos, A. 1942b. Los sedimentos prepampeanos del Valle del Nono (Sierra de Córdoba) Argentina. Publicaciones del Instituto de Fisiografía y Geología de la Universidad Nacional del Litoral, 13, 5-62.

Castellanos, A. 1944. Paleontología estratigráfica de los sedimentos neógenos de la provincia de Córdoba. Publicaciones del Instituto de Fisiografía y Geología de la Universidad Nacional del Litoral, 23, 5-47.

Castellanos, A. 1951. Un nuevo género de Esclerocaliptino ("Isolinia") descubierto en el arauncaniano del Valle de Los Reartes (Sierras de Córdoba). Revista de la Asociación Geológica Argentina, 6, 95-100.

Castellanos A. 1956. Rasgos geomorfológicos del valle de Los Reartes (Sierra de Córdoba). GAEA, 10, 21-56.

Castellanos, A. 1958. Nota preliminar sobre nuevos restos de mamíferos fósiles en el Brocherense del Valle de Los Reartes (provincia de Córdoba, Argentina). Congreso Geológico Internacional, 217-233.
Cattoi, N.V. 1966. Edentata. In: Paleontografía Bonaerense (ed. Borrello, A.V.), 2, 86-99.

Cione, A.L. \& Tonni, E.P. 1995. Bioestratigrafía y cronología del Cenozoico superior de la región pampeana. In: Evolución biológica y climática de la región pampeana durante los últimos cinco millones de años (eds. Alberdi, M.T., Leone, G. \& Tonni, E.P.). Un ensayo de correlación con el Mediterráneo occidental. Museo Nacional de Ciencias Naturales, Monografías, 12, 47-74.

Cope, E.D. 1889. The Edentata of North America. American Naturalist, 23, 657-664.

Croft, D.A., Flynn, J.J. \& Wyss, A.R. 2007. A new basal Glyptodontoid and other Xenarthra of the early Miocene Chucal Fauna, Northern Chile. Journal of Vertebrate Paleontology, 27, 781-797.

Cruz, L. E. 2011. La megafauna del Pleistoceno - Holoceno temprano de la Provincia de Córdoba y su comparación con la de Buenos Aires. Sistemática, Bioestratigrafía y Dinámica Faunística. PhD Thesis, Universidad Nacional de la Plata (unpublished).

Cruz, L. E. 2013. Biostratigraphy and geochronology of the late Cenozoic of Córdoba Province (central Argentina). Journal of South American Earth Sciences, 42, 250259.

de Jong, W.W., Zweers, A., Joysey, K.A., Gleaves, J.T. \& Boulter, D. 1985. Protein sequence analysis applied to Xenarthran and Pholidote phylogeny. In: The Evolution and Ecology of Armadillos, Sloths, and Vermilinguas. (ed. Montgomery, G.G.). Washington, DC. Smithsonian Institution Press, 65-76.

Delsuc, F., Scally, M., Madsen, O., Stanhope, M.J., de Jong, W.W. Catzeflis, F.M., Springer, M.S. \& Douzery, E.J.P. 2002. Molecular phylogeny of the living xenarthrans and the impact of character and taxon sampling on the placental tree rooting. Molecular Biology and Evolution, 19, 1656-1671.

Delsuc, F., Stanhope, M.J. \& Douzery, E.J.P. 2003. Molecular systematic of armadillos (Xenarthra, Dasypodidae): contribution of maximum likelihood and Bayesian analyses of the mitochondrial and nuclear genes. Molecular Phylogenetics and Evolution, 28, 261-275.

Fariña, R.A. 1985. Some functional aspects of mastication in Glyptodontidae. Fortschritte del Zoologie, 30. 277-280.

Fariña, R.A. 1993. Análisis filogenético de Glyptodontia. Ameghiniana, 30, 329-330.

Fariña, R.A. 2001. Física y Matemáticas para reconstruir la vida en el pasado. Actas de Fisiología, 6, 45-70.

Fariña, R.A. \& Vizcaíno, S.F. 2001. Carved teeth and strange jaws: How glyptodonts masticated. In: Biomechanics and Paleobiology of Vertebrates (eds. Vizcaíno, S.F., Fariña, R.A \& Janis, C.). Acta Paleontologica Polonica (Special Issue), 46, 87-102.

Ferigolo, J. 1985. Evolutionary trends in the histological pattern in the teeth of Edentata (Xenarthra). Archives of Oral Biology, 30, 71-82.

Fernicola, J.C. 2005. Análisis filogenético de la familia Glyptodontidae Gray. Ameghiniana, 42, 27. 
Fernicola, J.C. 2008. Nuevos aportes para la sistemática de los Glyptodontia Ameghino 1889 (Mammalia, Xenarthra, Cingulata). Ameghinana, 45, 553-574.

Fernicola, J.C. \& Porpino K.O. 2012. Exoskeleton and Systematics: A Historical Problem in the Classification of Glyptodonts. Journal of Mammalian Evolution, 19, 1-13.

Gaido, M.F., Murra, J.A.F. \& Sapp, M. 2006. Hoja Geológica 3166-36, Valle de Calamuchita, 1:100.000. Instituto de Geología y Recursos Minerales. Boletín No 361.

Gaudin, T.J. 1999. The morphology of xenarthrous vertebrae (Mammalia, Xenarthra). Fieldiana, 41, 1-38.

Gray, J. E. 1869. Catalogue of carnivorous, pachydermatous and edentates Mammalia in the British Museum. British Museum of Natural History, 1-398.

Hoffstetter, R. 1958. Xenarthra. In: Traité de Paléontologie (ed. Piveteau, J.), 6, 535-636.

Hoffstetter, R. 1982. Les edentés xenarthres, un groupe singulier de la faune néotropicale (origine, affinités, radiation adaptative, migrations et extinctions). In: Palaeontology, Essential of Historical Geology (ed. Montanaro Galitelli, E.). Modena, 383-443.

Huxley, T.H. 1864. On the osteology of the genus Glyptodon. Proceedings of the Royal Society of London, 13, 108.

Illiger, J.K.W. 1811. Prodromus systemalis mammalium et avium additis terminis zoographicis utriusque classis. Berlin, 1-301.

Kraglievich, J.L. 1934. La antigüedad de las faunas de Monte Hermoso y Chapadmalal, deducidas de su comparación con las que le precedieron y sucedieron. Imprenta "El Siglo Ilustrado”.

Marshall, L., Berta, A., Hoffstetter, R., Pascual, R., Reig, O.A., Bombin, M. \& Mones, A. 1984. Mammals and Stratigraphy: geochronology of the continental mammalbearing Quaternary of South America. Paleovertebrata, Memoire Extraordinaire.

McDonald, H.G. 2003. Xenarthran skeletal anatomy: primitive or derived (Mammalia, Xenarthra). Senckenbergiana Biologica, 83, 5-17.

McKenna, M.C. \& Bell, S.K. 1997. Classification of mammals above the species level. Columbia University Press, New York.

Moreira, L.E. 1965. Notas previas sobre nova espécie de mamífero fóssil do Estado do Caerá, Brasil. Hy Hy Té, 2, 41-49.

Moreira, L.E. 1971. Os gliptodontes do Nordeste do Brasil. Anais da Academia Brasileira de Ciencias, 43, 529552.

Patterson B., Segall W. \& Turnbull W.D. 1989. The ear region in xenarthrans (= Edentata, Mammalia). Part I. Cingulates. Fieldiana, 18, 1-46.

Paula Couto, J.C. 1979. Tratado de Paleomastozoología. Academia Brasileira de Ciencias.

Porpino, K.O. \& Bergqvist, L.P. 2002. Novos achados de Panochthus (Mammalia, Cingulata, Glyptodontoidea) no Nordeste do Brasil. Revista Brasileira de Paleontologia, 4, 51-62.
Porpino, K.O., Fernícola, J.C. \& Bergqvist, L.P. 2009. A new cingulate (Mammalia: Xenarthra) Pacharmatherium brasiliensis sp. nov., from the late Pleistocene of the Northeastern Brazil. Journal of Vertebrate Paleontology, 29, 881-893.

Porpino, K.O., Fernícola, J.C. \& Bergqvist, L.P. 2010. Revisting the intertropical brazilian species Hoplophorus euphractus (Cingulata, Glyptodontoidea) and the phhylogenetic affinities of Hoplophorus. Journal of Vertebrate Paleontology, 30, 911-927.

Redford, K.H. \& Eisenberg, J.F. 1992. Mammals of the Neotropics. The Southern Cone. University of Chicago, Chicago.

Sarich, V.M. 1985. Xenarthran systematics: albumin immunological evidence. In: The Evolution and Ecology of Armadillos, Sloths, and Vermilinguas (ed. Montgomery, G.G.). Smithsonian Institution Press, 77-81.

Scillato-Yané, G.J. 1976. Sobre un Dasypodidae (Mammalia, Xenarthra) de Edad Riochiquense (Paleoceno Superior) de Itaboraí, Brasil. Anales de la Academia Brasilera de Ciências, 48, 529-530.

Scillato-Yané, G.J. 1982. Los Dasypodidae (Mammalia, Edentata) del Plioceno y Pleistoceno de Argentina. $\mathrm{PhD}$ Thesis, Universidad Nacional de la Plata (unpublished).

Scillato-Yané, G.J. \& Carlini, A.A. 1995. Estado actual del estudio de los Xenarthra. Id., Simposio "Estado actual del estudio de los Xenarthra”. Abstract Book, p. 27.

Scillato-Yané, G.J., Carlini, A.A., Vizcaíno, S.F. \& OrtizJaureguizar, E. 1995. Xenarthra. In: Evolución biológica y climática de la región Pampeana durante los últimos cinco millones de años. Un ensayo de correlación con el Mediterraneo Occidental. (eds. Alberdi, M.T., Tonni, E.P. \& Leone, G.). Monografías de la CSIC, España, 183-209.

Scillato-Yané, G.J. \& Carlini, A.A. 1998. Un gigantesco gliptodonte en los alrededores de la Ciudad de La Plata. Revista Museo de La Plata, 11, 45-48.

Soibelzon, L.H., Zamorano, M., Scillato-Yané, G.J., Piazza, D., Rodríguez, S., Soibelzon, E., Tonni, E.P., San Cristóbal, J. \& Beilinson, E. 2012. Un glyptodontidae de gran tamaño en el Holoceno temprano de la región Pampeana. Revista Brasileira de Paleontologia, 15, 113-122.

Tauber, A.A. \& Álvarez, D. 2003. Mamíferos, paleoambientes y tafonomía de la Formación Brochero (Mioceno tardío-Plioceno) del valle de Nono, Córdoba, Argentina. Ameghiniana, 40, Suplemento Resúmenes, 108.

Wetzel, R.M. 1982. Systemathcs, distribution, ecology, and conservation of South American edentates. Special publication pymatuning laboratory of ecology, 6, 345375.

Wetzel, R.M. 1985. The Identification and Distribution of Recent Xenarthra (=Edentata). In: Ecology of Armadillos, Sloths, and Vermilinguas (ed. Montgomery, G.G.). Washington and London, Smthsonian Institution Press, 5-21.

Zamorano, M. 2005. Las especies de Xenarthros de la Región Pampeana comunes a las edades Ensenadense 
y Bonaerense. Bienal de Ciencia y Tecnología. Área Biología y Ecología. Abstract Book, in digital format.

Zamorano, M. \& Scillato-Yané, G.J. 2006. Descripción comparativa del cráneo e Panochthus cf. Intermedius Lydekker (Glyptodontidae, Hoplophorinae, Panochthini), del Ensenadense de la región pampeana. XXII Jornadas Argentinas de Paleontología de Vertebrados. Abstract Book, p. 36.

Zamorano, M., Scillato-Yané, G.J., González Ruiz, L.R. \& Zurita, A.E. 2011. Revisión de los géneros Nopachtus Ameghino y Phlyctaenopyga Cabrera (Xenarthra, Glyptodontidae, Hoplophorinae) del Mioceno tardío y Plioceno de Argentina. Revista del Museo Argentino de Ciencias Naturales "Bernardino Rivadavia”, 13, 59-68.

Zamorano, M. 2012a. Los Panochthini (Xenarthra, Glyptodontidae): Sistemática y evolución. PhD Thesis, Universidad Nacional de la Plata (unpublished).

Zamorano, M. 2012b. Los Panochthini (Xenarthra, Glyptodontidae): Sistemática y evolución. Mastozoología Neotropical, 19, 382-384.
Zamorano, M. \& Brandoni, D. 2012. Análisis filogenético de los "Panochthini” (Glyptodontidae, "Holplophorinae”). XXVI Jornadas Argentinas de Paleontología de Vertebrados. Abstract Book, in digital format.

Zamorano, M. \& Brandoni, D. en prensa. Phylogenetic analysis of the Panochthini (Xenarthra, Glyptodontidae, "Holplophorinae"). Alcheringa.

Zurita, A.E. 2007. Sistemática y evolución de los Hoplophorini (Xenarthra, Glyptodontidae, Hoplophorinae. Mioceno tardío-Holoceno temprano). Importancia bioestratigráfica, paleobiogeográfica y paleoambiental. PhD Thesis, Universidad Nacional de la Plata (unpublished).

Zurita, A.E., Zamorano, M., Scillato-Yané, G.J., GonzálezRuiz, L.R., Rivas Durán, B \& Céspedez Paz, R. 2011. An exceptional Pleistocene specimen of Panochthus Burmeister (Xenarthra, Glyptodontoidea) from Bolivia: its contribution to the understanding of the early-middle Pleistocene Panochthini. Comptes rendus Palevol, 10, 655-664. 